\title{
Hematopoietic Stem Cell in Acute Myeloid Leukemia Development
}

\author{
Sérgio Paulo Bydlowski and Felipe de Lara Janz \\ University of Sao Paulo School of Medicine \\ Laboratory of Genetics and Molecular Hematology, São Paulo, SP, \\ Brazil
}

\section{Introduction}

Hematopoietic stem cells (HSCs) are multipotent stem cells defined by their ability to selfrenewal, differentiation and maintenance of all blood cell types in the hematological system during the entire lifetime of the organism. This physiological process, called hematopoiesis, is controlled by several complex interactions between genetic processes in blood progenitor cells and bone marrow microenvironment. Hemostasis is maintained by a delicate balance between processes such as self-renewal, proliferation and differentiation versus apoptosis and cell-cycle arrest in HSCs. Over the last two decades, several studies have been made to understand possible mechanisms of cell malignancy and tumor growth, both in solid and hematological cancers. Leukemias are hematological malignancies that arise from cancer stem cells (CSCs). Neoplastic transformation of hematopoietic stem or progenitor cells occurs by unbalanced critical mechanisms. Blood cancers, as acute myeloid leukemia (AML), are sustained by leukemic stem cells (LSCs) which, like normal HSCs, present a range of biological characteristics that enable their long-term survival. AML is a well studied hematological cancer type characterized by an accumulation of clonal myeloid progenitor cells that do not differentiate normally. However, there is still no consensus about the mechanisms by which the HSCs transformation occurs. In this chapter, the hematopoietic stem cells and leukemic stem cells will be focused on leukemia development, mainly in AML.

\section{Hematopoietic stem cells}

Hematopoietic stem cells are a well characterized stem cell type which has been used in bone marrow transplantation for treatment of hematological malignancies as well as nonmalignant disorders (Warner et al, 2004). In fact, bone marrow (BM) has been, for many years, transplanted as an unfractionated cell pool, until researchers discovered which cellular components were responsible for the engraftment of the donor hematopoietic and immune systems in marrow-ablated patients.

HSCs present self-renewal potential and differentiation capacity into blood lineages. The self-renewal concept means that when stem cells divide, $50 \%$ of the daughter cells, on average, is committed with a cell lineage; the remaining $50 \%$ do not differentiate; therefore 
the process maintains the same number of stem cells. This is accomplished by the so-called asymmetric cell division, so that each dividing stem cell originates one new stem cell and one differentiated cell (Gordon, 2005) (Figure 1). In the symmetric division, the stem cells originate $100 \%$ of identical stem cells.

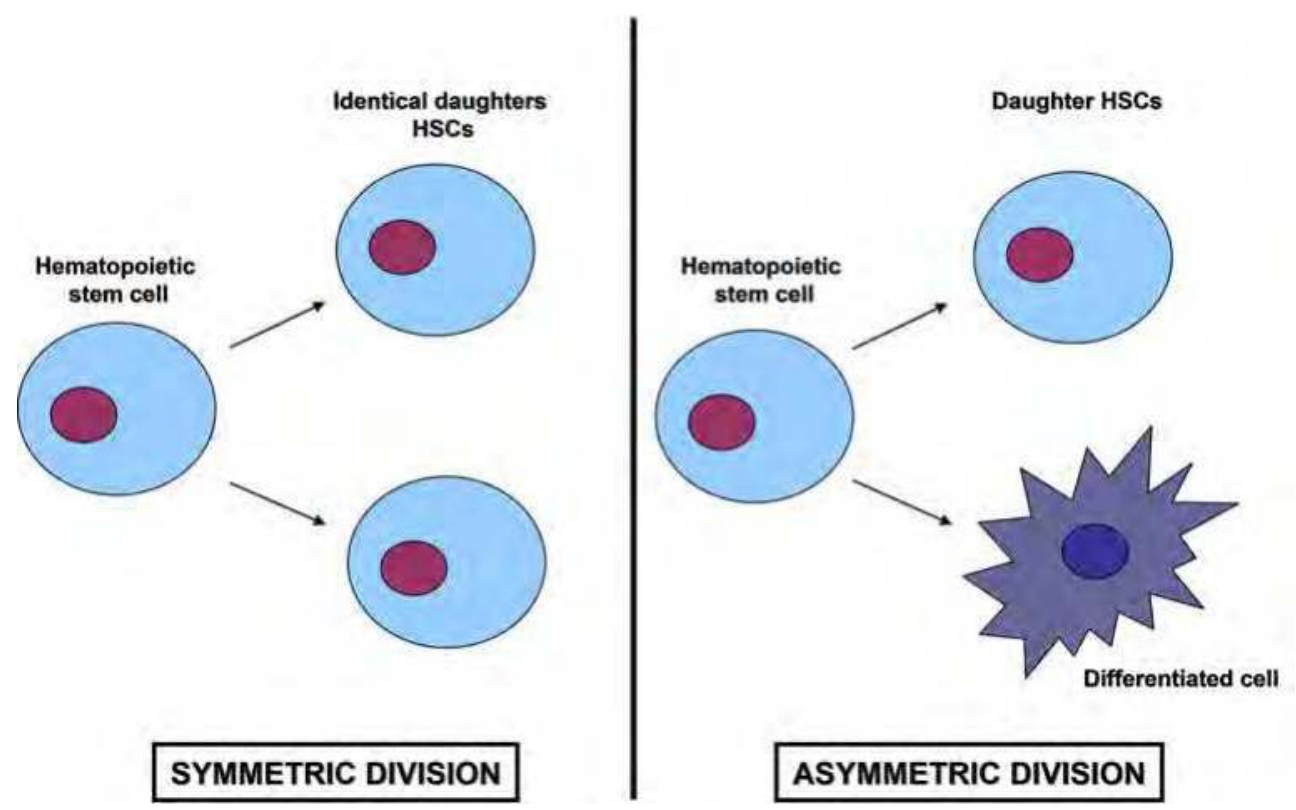

Fig. 1. Schematic illustration of two different types of HSCs division. On the left, the symmetric division in which mitosis originates two identical stem cell daughters. On the right, the characteristic stem cells asymmetric division where each dividing stem cell forms one new stem cell and one differentiated cell.

HSCs are classified as multipotent stem cells due to their ability to differentiate in lymphoid as well as myeloid cells types; however, some studies showed that transplanted bone marrow cells can contribute to the repair and regeneration of a spectrum of other tissue cell types including those from brain, muscle, lung and liver.

Lymphoid cell lineage includes $\mathrm{T}$ and $\mathrm{B}$ cells, while megakaryocytes, erythrocytes, granulocytes and macrophages belong to the myeloid lineage. These two lineages derive from different progenitor cells. Common lymphoid progenitors (CLPs) can differentiate into all types of lymphocytes without noticeable myeloid potential under physiological conditions. Similarly, common myeloid progenitors (CMPs) can give rise to all classes of myeloid cells with no or extensively low levels of B-cell potential (Kondo, 2010). It is likely that differences in the expression levels of transcription factors determine the lineage affiliation of a differentiating cell (Figure 2). The transcription factors PU.1 and GATA-1 have been implicated in myeloid and erythroid/megakaryocyte lineage differentiation, respectively (Gordon, 2005). 


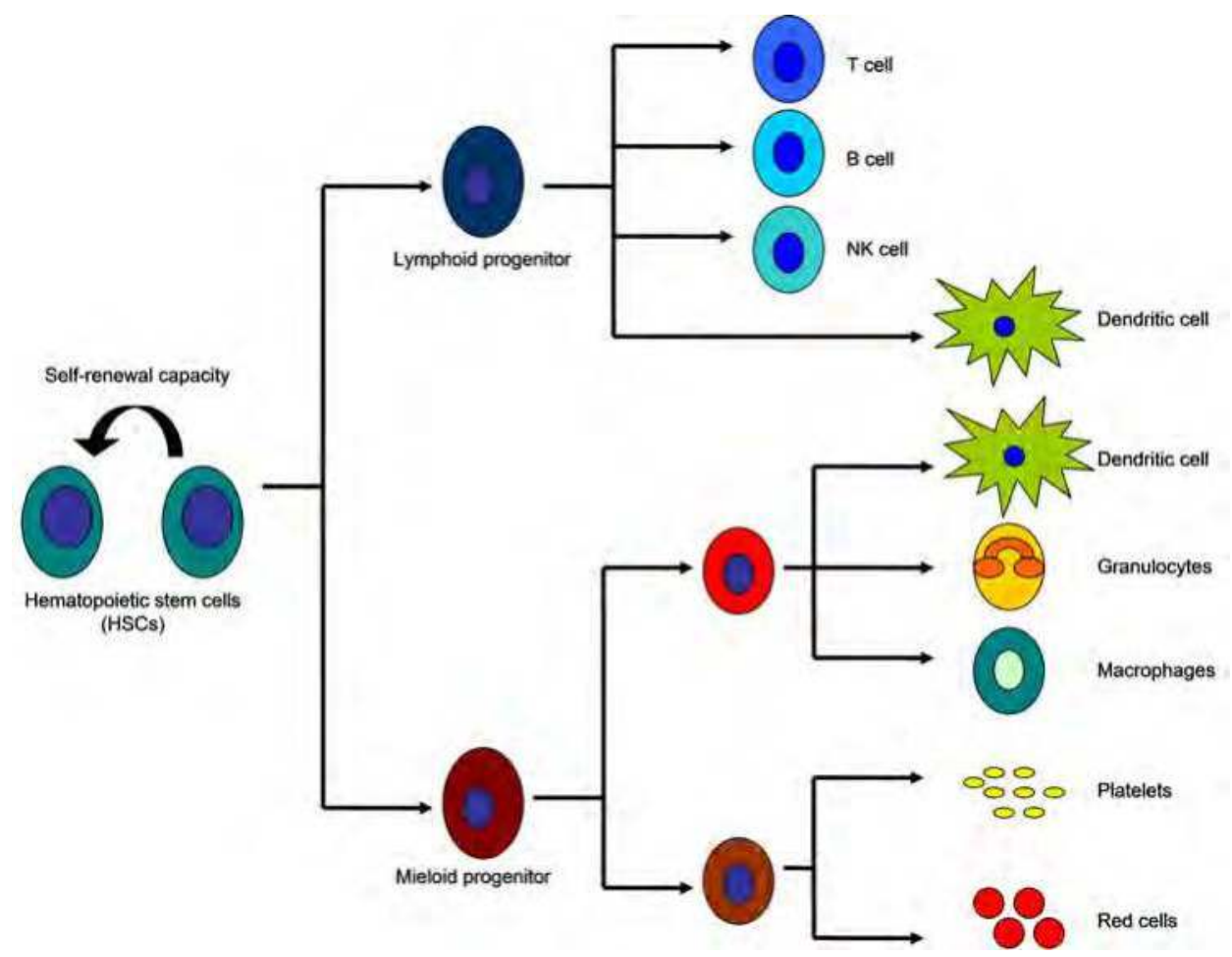

Fig. 2. HSCs differentiation pathways. HSCs could differentiate into specific lymphoid and myeloid cell types. Common lymphoid progenitors (CLPs) can differentiate into all types of lymphocytes and common myeloid progenitors (CMPs) can give rise to all classes of myeloid cells (megakaryocytes, erythrocytes, granulocytes and macrophages) (Adapted from Du et al., 2008).

\subsection{Characterization}

Morphologically, hematopoietic stem cells are undifferentiated and resemble small lymphocytes. Normally, a large fraction is quiescent, in the $\mathrm{G}_{0}$ phase of the cell cycle, which protects them from the action of cell cycle-dependent drugs. The quiescent state of stem cells is maintained by transforming growth factor- $\beta$ (TGF- $\beta$ ). The activity of TGF- $\beta$ is mediated by $\mathrm{p} 53$, a tumor suppressor gene that regulates cell proliferation and targets the cyclindependent kinase inhibitor p21 (Gordon, 2005). Quiescence of HSCs is critical not only for protecting the stem cell compartment and sustaining stem cell pools during long periods of time, but also by minimizing the accumulation of replication-associated mutations.

Quiescence regulation in HSCs is also of great importance for understanding the pathophysiological origins of many related disorders. Interestingly, many of the intrinsic transcriptional factors that maintain HSCs quiescence are found to be associated with leukemias. For example, chromosomal translocations resulting in the fusion of FoxOs and myeloid/lymphoid or mixed lineage leukemia have been reported in acute myeloid leukemias. 
The majority of normal HSCs are present among the CD34+/CD38- bone marrow cell fractions; some HSCs are also observed among CD34-/Lin- cells; CD34+/CD38+ cell fractions contain some HSCs but endowed with short-term repopulating activity. Other recognized marker is the tyrosine kinase receptor c-kit (CD117), concomitantly with the lack of terminal differentiation markers (as CD4 and CD8; Figure 3) (Rossi et al., 2011).

Primitive HSCs populations show low fluorescence ratios after Hoechst 33342 and Rhodamine 123 staining; these cells are described as side population (SP). SP cells demonstrate high expression of ATP binding cassette $(\mathrm{ABC})$ transporters as P-glycoprotein (P-gp/ABCB1), breast cancer resistance protein (BCRP/ABCG2) and lung resistance protein (LRP) (Huls et al., 2009). MDR1 has been implicated in the protection of cells against apoptotic cell death induced by a variety of methods including growth factor deprivation, UV irradiation, ionizing radiation, or tumor necrosis factor-a treatment. BCRP is a halftransporter and characterized as a novel stem cell transporter. Like MDR1, enforced overexpression of BCRP in human MCF-7 breast cancer cells confers a broad spectrum of drug resistance, and elevated levels of expression of BCRP have been reported to be associated with acute myeloid leukemia.

Since ABC transporter function is associated with both normal and aberrant hematopoiesis, it is important to fully characterize the function of this class of transporter proteins in hematopoietic cell differentiation and to define the underlying mechanisms.

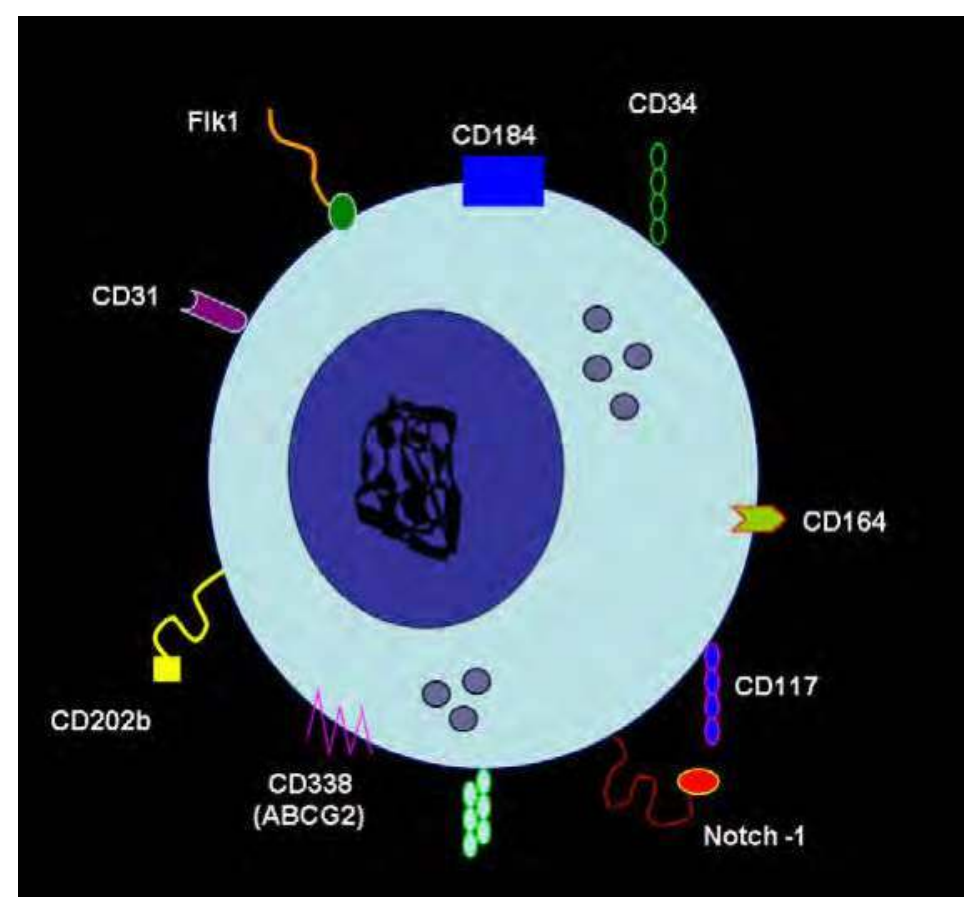

Fig. 3. HSCs main surface markers. HSCs express typical antigens as: CD34, CD117, CD164, CD202b, CD31, Flk-1, CD184, CD338 or ABCG2, Notch-1 concomitantly with the lack of terminal differentiation markers (CD4 and CD8). 


\subsection{Classification}

According to its hematopoietic repopulation capacity, the hematopoietic stem cell pool can be subdivided into three main groups:

a. short-term HSCs, capable of generating clones of differentiating cells for only 4-6 weeks;

b. intermediate-term HSCs, capable of sustaining a differentiating cell progeny for 6-8 months before becoming extinct;

c. long-term HSCs, capable of maintaining hematopoiesis indefinitely (Testa, 2011).

\subsection{HSC sources}

HSCs can be harvested from healthy donors either by bone marrow aspiration, peripheral stem cell mobilization or from umbilical cord blood (Dick, 2003). HSCs located in the bone marrow present an estimated frequency of $0.01 \%$ of total nucleated cells and can be collected by iliac crest puncture and then separated from the other blood cells by magnetic beads or cell sorting.

Umbilical cord blood (UCB) is a source of the rare but precious primitive HSCs and progenitor cells that can reconstitute the hematopoietic system in patients with malignant and nonmalignant disorders treated with myeloablative therapy. UCB cells possess an enhanced progenitor cell proliferation capacity and self-renewal in vitro. UCB is usually discarded and it exists in almost limitless supply. The blood remaining in the delivered placenta is safely and easily collected and stored. The predominant collection procedure currently involves a relatively simple venipuncture, followed by gravity drainage into a standard sterile anti-coagulant-filled blood bag, using a closed system, similar to that utilized for whole blood collection (Bojanic \& Golubic Cepulic, 2006).

Peripheral blood hematopoietic stem cells (PBSCs) have numerous advantages in comparison with traditionally used bone marrow. PBSCs collection by leukapheresis procedure is simple and better tolerated than bone marrow harvest. PBCSs are mobilized by myelosupressive chemotherapy or/and hematopoietic growth factors. Leukapheresis product contains PBSCs along with committed lineage of progenitors and precursors which contribute to faster hematopoietic recovery.

Unfortunately, the expansion of HSCs in vitro is difficult to achieve because the proliferation is accompanied by differentiation. This is presumably caused by a lack of appropriate cues that are provided in vivo by the microenvironment. The most excellent defined culture medium for HSCs expansion is supplemented with cytokines such as fetal liver tyrosine kinase-3 ligand (FLT3-L), stem cell factor (SCF), interleukin-3 (IL-3) and thrombopoietin (TPO). Interestingly, mesenchymal stem cells (MSCs), which are characterized by multi-differentiation potential, are important players of the bone marrow HSCs niche. In recent years, MSCs have been shown to support HSCs maintenance and engraftment (Jing et al., 2010).

\section{Factors involved in hematopoiesis}

Hematopoiesis is a highly coordinated process wherein HSCs differentiate into mature blood cells supported by a physical environment called niche (Figure 3). The bone marrow 
niche is the most important post-natal microenvironment in which HSCs proliferate, mature and give rise to myeloid and lymphoid progenitors. BM is present in the medullary cavities of all animal bones. Unlike secondary lymphoid organs such as spleen with distinct gross structures including red and white pulp, BM has no clear structural features, except for the endosteum that contains osteoblasts. The endosteum region comes in contact with calcified hard bones and provides a special microenvironment to HSCs, which is necessary for the maintenance of HSC activity (Kondo, 2010).

Within the niche, HSCs are believed to receive support and growth signals originating from several sources, including: fibroblasts, endothelial and reticular cells, adipocytes, osteoblasts and mesenchymal stem cells. The main function of the niche is to integrate local changes in nutrients, oxygen, paracrine and autocrine signals and to change HSCs quiescence, trafficking, and/or expansion in response to signals from the systemic circulation (Broner \& Carson, 2009). Although the nature of true MSCs remains misunderstood, CXC chemokine ligand 12 (CXCL12) - expressing CD146 MSCs were recently reported to be self-renewing progenitors that reside on the sinusoidal surfaces and contribute to organization of the sinusoidal wall structure, produce angiopoietin-1 (Ang-1), and are capable of generating osteoblasts that form the endosteal niche (Konopleva \& Jordan, 2011).

These CXCL12 reticular cells may serve as a transit pathway for shuttling HSCs between the osteoblastic and vascular niches where essential but different maintenance signals are provided. Cytokines and chemokines produced by bone marrow MSCs concentrate in particular niches secondary to varying local production and through the effects of cytokinebinding glycosaminoglycans. Of these, CXCL12/stromal cell-derived factor-1 alpha positively regulates HSCs homing, while transforming growth factors FMS-like tyrosine kinase 3 (Flt3) ligand and Ang-1 function act as quiescence factors.

CXCL12-CXCR4 signaling is involved in homing of HSCs into BM during ontogeny as well as survival and proliferation of colony-forming progenitor cells. The CXCR4-selective antagonist-induced mobilization of HSCs into the peripheral blood further indicates a role for CXCL12 in retaining HSCs in hematopoietic organs. BM engraftment involves subsequent cell-to-cell interactions through the BMSC-produced complex extracellular matrix. Thus, vascular cell adhesion molecule-1 (VCAM-1) or fibronectin is critical for adhesion to the BM derived MSCs.

In this way, the control of hematopoietic stem cell proliferation kinetics is critically important for the regulation of correct hematopoietic cells production. These control mechanisms could be classified in intrinsic or extrinsic to the stem cells, or a combination of both.

Extrinsic control means that self-renewal and differentiation can be controlled by external factors, such as cell-cell interactions in the hematopoietic microenvironment or cytokines as SCF (stem cell factor) and its receptor c-kit, Flt-3 ligand, TGF- $\beta$, TNF- $\alpha$ and others. Cytokines regulate a variety of hematopoietic cell functions through the activation of multiple signal transduction pathways. The major pathways relevant to cell proliferation and differentiation are the Janus kinase (Jak)/signal transducers and activators of transcription (STATs), the mitogen-activated protein (MAP) kinase and the phosphatidylinositol (PI) 3-kinase pathways. 
Yet, in intrinsic control, the expression of other transcription factors has been shown to be essential for hematopoietic cell development from the earliest stages, as: SCL (stem cell leukaemia hematopoietic transcription factor); GATA-2; gene products involved in cell cycle control, such as the cyclin dependent kinase inhibitors (CKIs) p16, p21 and p27.

Notch-1-Jagged pathway may serve to integrate extracellular signals with intracellular signalling and cell cycle control. Notch-1 is a surface receptor on hematopoietic stem cell membranes that binds to its ligand, Jagged, on stromal cells. This results in cleavage of the cytoplasmic portion of Notch-1, which can then act as a transcription factor (Gordon, 2005).

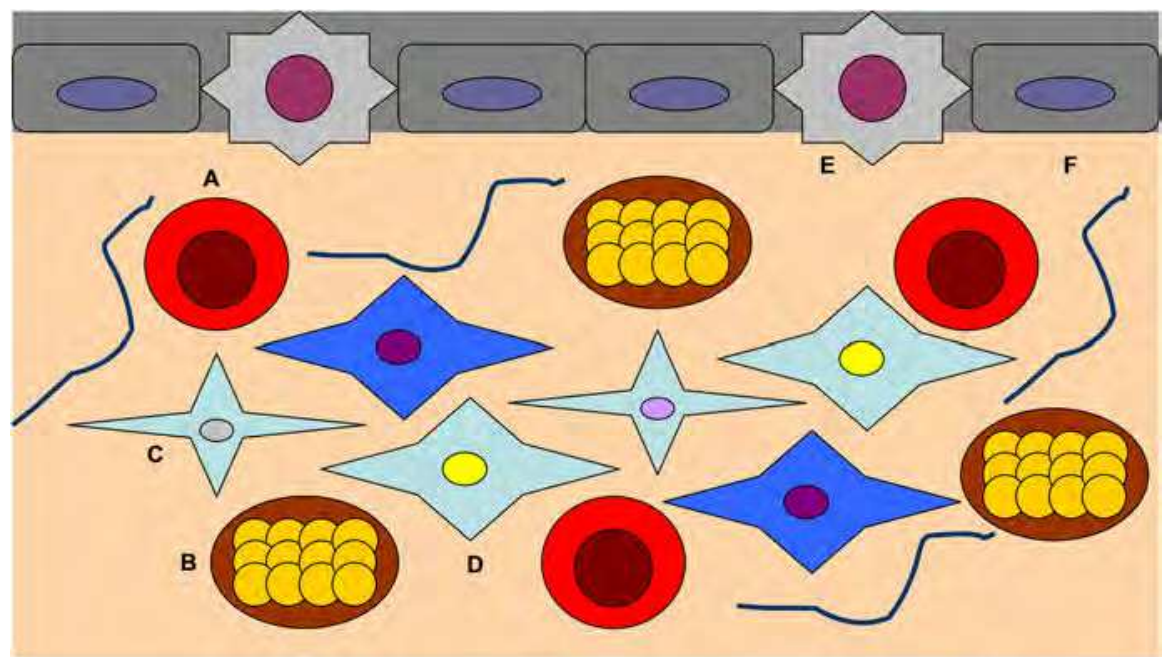

Fig. 4. Schematic representation of main bone marrow niche cells: A- hematopoietic stem cells, B- adipocytes, C- mesenchymal stem cells, D- reticular cells, E- osteoclasts, and Fosteoblasts.

\section{Leukemia and leukemic development}

Leukemia is the consequence of stepwise genetic alterations that confer both proliferative and survival advantage, as well as self-renewal capacity to the malignant cells (Lane et al., 2011). When the HSCs processes of self-renewal and differentiation become deregulated or uncoupled, leukemias can result, characterized by an accumulation of immature blast cells that fail to differentiate into functional cells. Two types of abnormal events can lead to leukemia. First, a normal stem cell acquires several mutations (from different types of genetic events) and, due to epigenetic changes that alter its growth control, the resistance to apoptosis is increased, interfering with the ability of its progeny to differentiate. Second, partially differentiated cells restore gene expression patterns that allow them to reacquire the unique self-renewal properties of stem cells while also interferes with their subsequent ability to differentiate (Testa, 2011).

Hanahan \& Weinberg (2000) described the rules that govern the transformation of normal cells into a malignant cell. The six main properties that define malignant cells are: selfsufficiency in growth signals; insensitivity to growth inhibitory signals; evasion of 
programmed cell death (apoptosis); limitless replicative potential; sustained angiogenesis; and tissue invasion and metastasis.

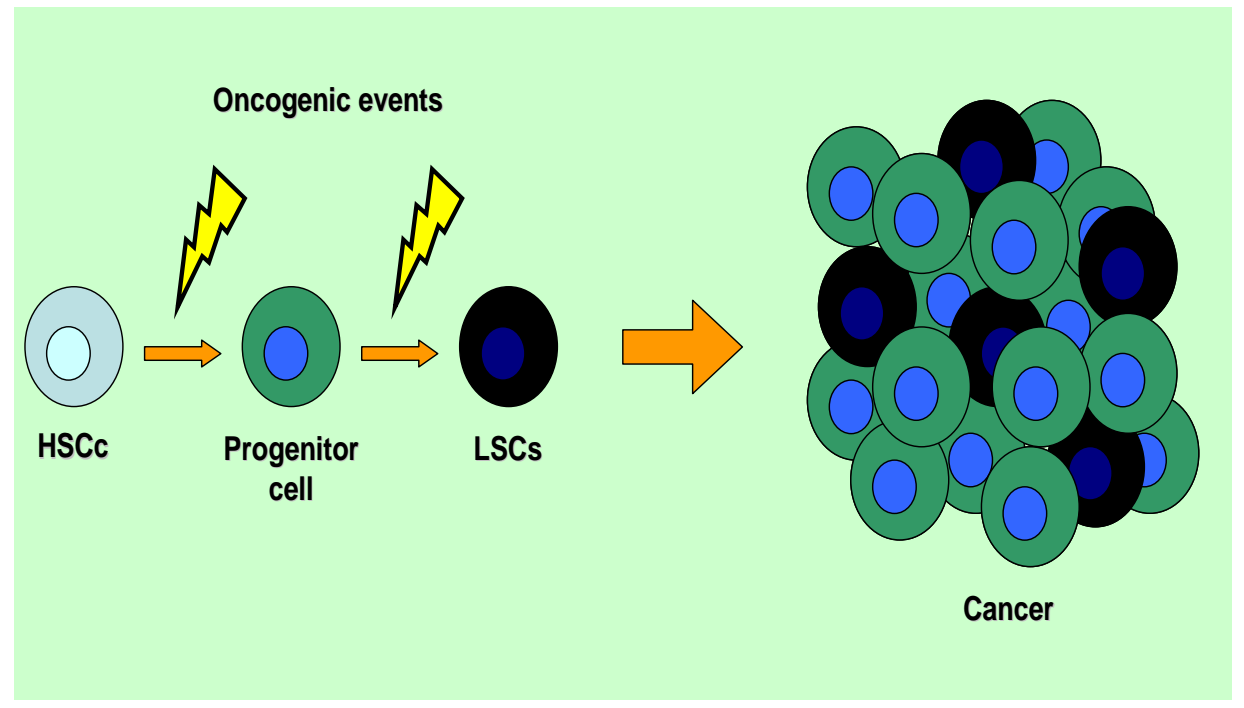

Fig. 5. Cancer stem cell hypothesis. A normal stem cell acquires several mutations and in consequence, by epigenetic changes that alter its growth control, its resistance to apoptosis increased and the ability of its progeny to differentiate is changed. Partially differentiated cells restore gene expression patterns that allow these cells to reacquire the unique self-renewal properties of stem cells while also interfere with their subsequent ability to differentiate.

The "cancer stem cell hypothesis" has gained considerable interest in recent years. This theory states that cells in a tumor are organized as a hierarchy similar to that of normal tissues, and are maintained by a small subset of tumor cells that are ultimately responsible for tumor formation and growth. These cells, defined as "cancer stem cells" (CSCs) or "tumor initiating cells" (TICs), possess several key properties of normal tissue stem cells including self-renewal, unlimited proliferative potential (i.e., the ability of a cell to renew itself indefinitely in an undifferentiated state), infrequent or slow replication, resistance to toxic xenobiotics, high DNA repair capacity, and the ability to give rise to daughter cells that differentiate. However, the major difference between cancer growth and normal tissue renewal is that whereas normal transit amplifying cells usually differentiate and die, at various levels of differentiation, the cancer transit-amplifying cells fail to differentiate normally and instead, accumulate (i.e. they undergo maturation arrest), resulting in cancer growth (Soltanian \& Matin, 2011).

In the last years, studies have also clearly demonstrated that leukemia populations are highly heterogeneous and that the disease is propagated by a subpopulation of leukemia stem cells (LSC). LSCs, like normal hematopoietic stem cells, possess a range of biological characteristics that enable their long-term survival. Therefore, LSCs reside in a mostly quiescent state, and as a consequence, the overall activity of many chemotherapeutic agents that function by targeting cycling cells is likely diminished (Konopleva \& Jordan, 2011). 
LSCs infiltrate the bone marrow and interfere with the normal HSC-microenvironment homeostasis. Available data indicate that LSCs also interact with the hematopoietic microenvironment to maintain self-renewal and to mitigate the effects of cytotoxic chemotherapy (Lane et al., 2011)

\subsection{Leukemia stem cells characterization}

The immunophenotype and isolation of LSCs were first described by Lapidot et al.(1994) from primary human AML samples and, later, studies have shown that LSCs can be defined as expressing CD34, CD382, HLA-DR2, CD902, CD117 and CD123. Some of these markers are also detected in HSCs, but the expression of CD123 seems to be leukemic-specific (Blair et al., 1998).

Another LSC specific antigen is C-type lectin-like molecule-1, CLL-1. This antigen was demonstrated to be capable of identifying residual leukemic CD34+CD38- cells in clinical remission bone marrow samples. However, more recent data indicate that the phenotype of LSCs may be somewhat variable from patient to patient and that, in some cases, more than one phenotypically distinct subpopulation may possess LSC activity (Konopleva \& Jordan, 2011).

Expression of Oct- 4 is another similarity between normal and cancer stem cells. Oct- 4 , a member of the family of POU-domain transcription factors, is expressed in pluripotent embryonic stem and germ cells. Oct- 4 mRNA is normally found in totipotent and pluripotent stem cells of pregastrulation embryos (Soltanian \& Matin, 2011). Expression of this factor plays a crucial role in maintaining the self-renewing, cancer stem-cell-like, and chemoradioresistant properties in lung cancer-derived CD133+ cells (Chen et al., 2008).

Oct-4 gene product is expressed in several types of adult pluripotent stem cells including kidney, breast, epithelial, pancreatic, mesenchymal, gastric and liver, as well as in tumor cell lines derived from pancreas and liver (Tai et al., 2005). According to Marques et al. (2010) it is also possible that the resistance phenotype developed by leukemic cells is determined by $A B C$ transporter expression which is probably activated by the induction of the Oct- 4 transcription factor. The ABCB1, ABCG2 and ABCC1 transporters exhibit binding sites (octamerATGCAAAT) for the Oct- 4 transcription factor. The presence of these binding sites in the gene promoter of these transport proteins suggests that the transporter regulation pathways may be initiated at the Oct- 4 recognized binding sites. However, the presence of Oct- 4 alone is not always sufficient for induction of transporter genes. Transporter expression levels are often dependent upon Oct-4 interactions with other transcription factors.

\subsection{Genetic pathways of LSCs}

\subsubsection{Wnt/Catenin}

The Wnt/Catenin signaling has been implicated in the self-renewal of LSCs. Wnt proteins are a large family of glycoproteins that bind to Frizzled receptors and LRP5/ 6 coreceptors. By stabilizing the mediator $\beta$-catenin, they start a complex signaling cascade that plays a significant role in regulating cell proliferation and differentiation. Wnt cascade has appeared as a critical regulator of stem cells self-renewal. Comparing the expression of normal hematopoietic stem cells to that of AML leukemic stem cells, evidences show that the Wnt signaling pathway is aberrantly regulated in leukemic stem cells. 


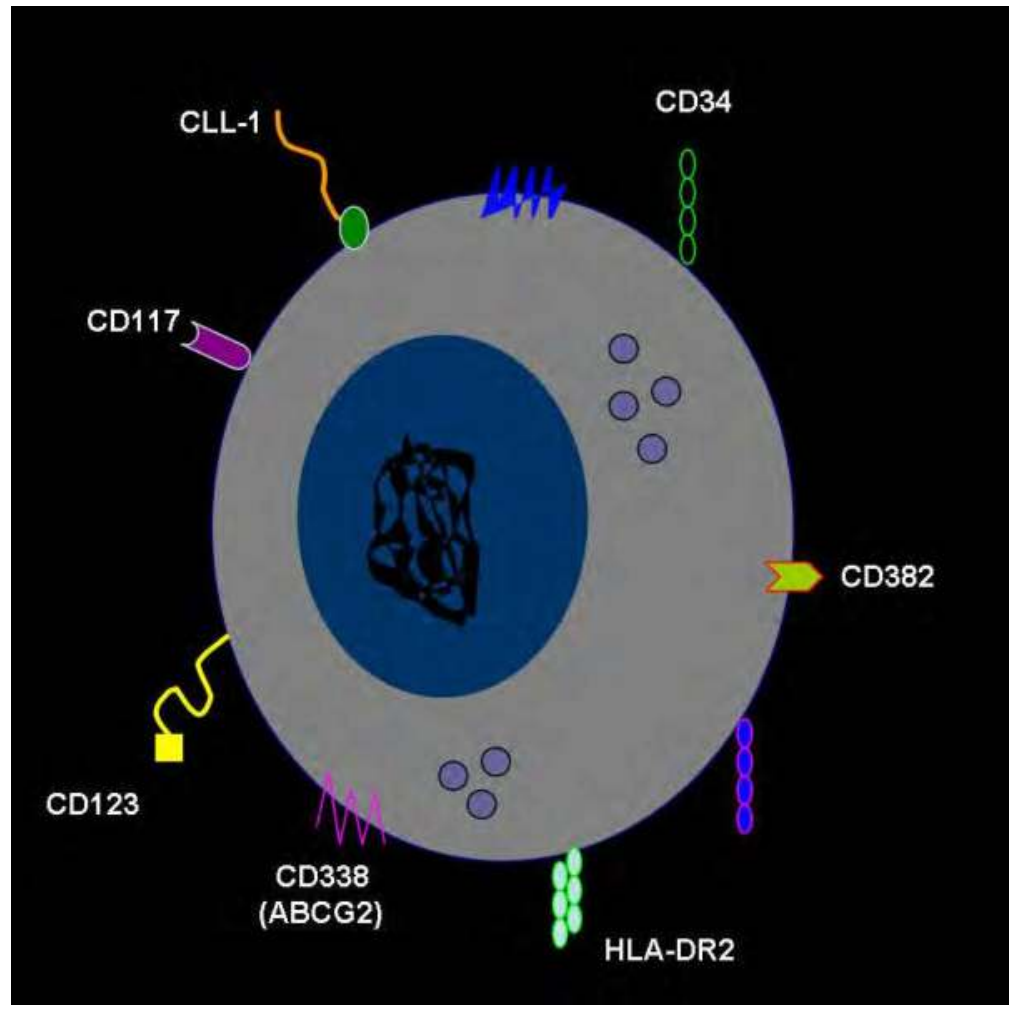

Fig. 6. LSCs main surface markers. LSCs can be defined as CD34, CD382, HLA-DR2, CD902, CD117 and CD123. The expression of CD123 seems to be leukemic-specific. Another LSC specific antigen is C-type lectin-like molecule-1, CLL-1.

\subsubsection{PTEN (Akt/mTOR)}

PTEN is a phosphatase that negatively regulates signaling through the PI3K pathway, attenuating proliferation and survival signals. PTEN deficiency causes an initial expansion of normal hematopoietic stem cells due to their cycling, followed by their exhaustion. In contrast to this requirement for PTEN in the maintenance of hematopoietic stem cells, leukemic stem cells arise and expand in numbers following PTEN deletion. The observation that PTEN deletion had opposite effects on normal hematopoietic stem cells compared to leukemic stem cells raised possibility for therapeutic targeting of this pathway to eliminate only the leukemic stem cells, without affecting normal hematopoietic stem cells. Since PTEN deletion causes increased AKT and mTOR activation, it seems logical that mTOR targeting by pharmacological agents, such as rapamycin, could represent an interesting option for AML treatment (Testa, 2011). 


\subsubsection{NF-kB}

In addition to genes involved in the control of stem cell self-renewal, leukemic stem cells are expected to express, at high levels, genes involved in anti-apoptotic mechanisms. In this context, particular attention has been focused on the study of NF-kB. NF-kB plays a critical role in inflammation, anti-apoptotic responses, and carcinogenesis. High NF-kB expression was found in primitive AML blasts. In particular, the constitutive activation of NF-kB was observed in AML cell populations enriched in leukemic stem cells, but not in normal hematopoietic stem cells. According to these observations, it seemed clear that NF-kB could be a potential therapeutic target for attempting leukemia stem cell eradication (Guzman et al., 2001).

\subsubsection{BMl1}

BMI1 is a polycomb group protein which, together with Ring1 proteins, is part of PRC1 complex that has histone H2A-K119 ubiquitin E3 ligase activity. BMI1 has a role in HOX gene (HOXC13) silencing by H2A ubiquitylation (Cao et al, 2005). BMI1 is also known to be important in the regulation and maintenance of proliferative/self-renewal potential in both normal hematopoietic and leukemic stem cells (Park et al, 2003). Upon knockdown of BMI-1, cells lose their ability to engraft and reconstitute leukemia in mice (Bomken et al., 2010).

\subsection{Xenotransplantation model of leukemia}

A key component for understanding the biological mechanisms for tumor heterogeneity is the ability to functionally assess the capacity for limitless proliferative capacity for segregated populations of tumor cells. Unfortunately, for hematologic malignancies, in vitro culture assays are not entirely effective as a means of functionally assessing self-renewal capacity. Thus, transplantation assays in which candidate populations are assessed for their ability to establish long-term serial engraftment of recipient animals is the gold standard for assigning limitless proliferative capability, i.e. self-renewal. For murine studies, the availability of syngeneic transplantation models has been responsible in large part for our in depth understanding of the normal murine hematopoietic hierarchy.

Since the $70 \mathrm{~s}$, there is ample evidence supporting the existence of a discrete compartment of slowly cycling leukemic cells that are resistant to standard chemotherapeutic agents. These cell populations were felt to represent the leukemic stem cells and though many observations were consistent with this hypothesis, there was no direct evidence that this was indeed the case. As the first direct evidence for the existence of cancer stem cells, the work of Lapidot et al. (1994) represented a milestone in the history of the leukemic stem cell model. This study identified an infrequent population of leukemic cells capable of recapitulating the human tumor in xenotransplants. A key finding was that the SCID mouse leukemia repopulating cell, SRC, possessed a phenotype that was similar to that of the normal hematopoietic stem cell (CD34+ and CD38-).

\subsection{Acute myeloid leukemia}

Acute myeloid leukemia (AML) is a clonal disorder characterized by arrest of differentiation in the myeloid lineage coupled with an accumulation of immature progenitors in the bone marrow, resulting in hematopoietic failure (Pollyea et al., 2011). AML is the most common 
acute leukemia in adults, affecting roughly three out of 100.000 people. AML patients are predominantly elderly, with a median age at diagnosis of 67 (National Cancer Institute 1975-2007).

In AML, there is wide patient-to-patient heterogeneity in the appearance of the leukemic blasts. Conventionally, AML is classified into seven French-American-British (FAB) subtypes corresponding to the maturation stage of the leukemia (Warner et al., 2004).

The discovery of leukemia-initiating cells in acute myeloid leukemias (AMLs) started with the discovery that the large majority of AML blasts do not proliferate and only a small minority is capable of forming new colonies (Testa, 2011).

A common feature to all AML cases is the arrested aberrant differentiation leading to an accumulation of more than $20 \%$ blast cells in the bone marrow (Gilliland \& Tallman, 2002). More than $80 \%$ of myeloid leukemias are associated with at least one chromosomal rearrangement (Pandolfi, 2001), and over 100 different chromosomal translocations have been cloned (Gilliland \& Tallman, 2002). Frequently, these translocations involve genes encoding transcription factors that have been shown to play an important role in hematopoietic lineage development. Thus, alteration of the transcriptional machinery appears to be a common mechanism leading to arrested differentiation (Pandolfi, 2001; Tenen, 2003).

Clinical investigation and experimental animal models suggest that at least two genetic alterations are required for the clinical manifestation of acute leukemia. According to the model proposed by Gilliland \& Tallman (2002), cooperation between class I activating mutations and class II mutations that induce termination of differentiation give rise to AML. The class I mutations, such as mutations in the receptor tyrosine kinase genes FLT3 and KIT, RAS family members, and loss of function of neurofibromin 1, confer proliferative and/or survival advantage to hematopoietic progenitors, typically as a consequence of aberrant activation of signal transduction pathways. The class II mutations lead to a halt in differentiation via interference with transcription factors or co-activators (Frankfurt et al., 2009).

While the LSC appears to share many of the cell surface markers previously identified for HSC such as CD34, CD38, HLA-DR, and CD71, there have been several groups who have reported surface markers that are differentially expressed in the two populations. CD90 or Thy- 1 is one marker that has been described to be potentially specific of the LSC compartment. Thy- 1 is downregulated in normal hematopoiesis as the most primitive stem cells progress toward the progenitor stage. This finding of the lack of expression on LSC might suggest that the primitive stem cell does not contribute to the primary pathological event, or that Thy-1 expression is downregulated as a result of the leukemogenic events (Hope et al., 2003).

The interaction between CXCL12 (stromal cell-derived factor-1 alpha) and its receptor CXCR4 on leukemic progenitor cells contributes to their homing to the bone marrow microenvironment. CXCR4 levels are significantly elevated in leukemic cells from patients with AML and CXCR4 expression is associated with poor outcome (Konopleva \& Jordan, 2011). Constitutive activation of the nuclear factor kappa B (NF-kB) pathway in primary human AML stem cells provided evidence that NF-kB plays a significant role in the overall survival of LSCs as well as AML cell types in general. This pathway is strongly implicated as a central target in developing LSC-specific therapies (Konopleva \& Jordan, 2011). 
FLT3, a member of the class III tyrosine kinase receptor family, is expressed in normal hematopoietic progenitors as well as in leukemic blasts, and it plays an important role in cell proliferation, differentiation, and survival. Activation of the FLT3 receptor by the FLT3 ligand leads to receptor dimerization and phosphorylation, and activation of downstream signaling pathways, including the Janus kinase (JAK) 2 signal transducer (JAK2), signal transducer and activator of transcription (STAT) 5, and mitogen-activated protein kinase (MAPK) pathways. Mutations in the FLT3 gene, found in approximately $40 \%$ of patients with AML, are believed to promote its autophosphorylation and constitutive activation, leading to ligand-independent proliferation (Frankfurt et al., 2009).

The adhesion receptor CD96 (TACTILE) is a transmembrane glycoprotein possessing three extracellular immunoglobulin-like domains. It is a member of the Ig gene superfamily and was first identified as a gene expressed in activated T cells. CD96 was described as a tumor marker AML stem cell (Konopleva \& Jordan, 2011). Hosen et al. (2011) showed that AMLLSC can be distinguished from normal HSC by the presence of CD96 expression. This finding suggests that CD96 also may prove to be an excellent target for antibody therapy against LSC because hematopoietic progenitors are regenerated rapidly from HSC.

Another adhesion molecule, CD44, has been demonstrated to be a key regulator of AML LSCs homing to microenvironmental niches, maintaining a primitive state. CD44 mediates adhesive cell-cell and cell-complex extracellular matrix interactions through binding to its main ligand, hyaluronan, a glycosaminoglycan highly concentrated in the endosteal region. Other ligands include osteopontin, fibronectin, and selectin, all of which are involved in cell trafficking and lodgment. Beyond its adhesion function, CD44 can also transduce multiple intracellular signal transduction pathways when bound to hyaluronan or to specific function-activating monoclonal antibodies (Konopleva \& Jordan, 2011).

\section{Conclusions}

Adult hematopoietic stem cells are undifferentiated cells capable of self-renewal and differentiation potential in several cell types that comprise the hematopoietic tissue. These cells have been used in bone marrow transplantation for treatment of hematological malignancies as well as non-hematological diseases. The HSC is the main component in the process of hematopoiesis which, together with the cells that make up the bone marrow stromal environment and other intrinsic and extrinsic factors, orchestrates the entire production of progenitors and terminally differentiated blood cells.

However, when this process of cell production is unbalanced, leading to an exacerbated and uncontrolled proliferation of blood progenitor cells, leukemia may develop.The ultimate challenge in coming years will be to understand the stem cell 'programme', particularly the control of self-renewal, in an attempt to develop novel, stem cell-directed therapies. An improved understanding of clonal evolution will be critical if we are to ensure that cancers are not able to evolve mechanisms to evade the new directed therapies. However, reducing the risk of relapse and minimizing long-term side effects should always remain the ultimate goal of understanding the CSCs.

With little doubt, the leukemia stem cell model has had the greatest clinical impact on our understanding and treatment of Philadelphia chromosome positive leukemias. Effective 
targeted agents and the ability to follow the impact of therapy on critical rare subpopulations of the malignant clone has greatly advanced our understanding of chronic myeloid leukemia.

For AML, the clinical impact of the leukemic stem cell model is less clear. The ability to isolate and characterize rare LSC populations has had a significant importance on our understanding of the biology of AML. In the past decade, we have gained considerable insight into the properties that distinguish leukemic stem cells from their normal counterparts and some of the rules that govern the leukemic hierarchy.

Despite the wide variance in techniques and to some degree expression profiles, common signaling pathways have been shown to play a role not only in AML stem cells, but also in cancer stem cells in general. These include BMI1, Wnt, Sonic Hedgehog, Notch, and NF-kB. These pathways are being evaluated for their role in LSC biology and agents targeting these pathways are making their way through the pre-clinical focus.

\section{References}

Becker, M. W. \& Jordan, C. T. (2011). Leukemia stem cells in 2010: Current understanding and future directions. Blood Reviews Vol.25, No. 2, (March 2011), pp. 75-81.

Blair, A.;Hogge, D.E. \& Sutherland, H.J. (1998). Most acute myeloid leukemia progenitor cells with long-term proliferative ability in vitro and in vivo have the phenotype CD34p/CD71-/HLA-DR-. Blood Vol.92, No. 11, (December 1998), pp. 4325-4335.

Bojanić, I. \& Golubić Cepulić, B. (2006).Umbilical cord blood as a source of stem cells. Acta Medica Croatica Vol.60, No. 3, (June 2006),pp. 215-225.

Bomken, S., Fiser, K., Heidenreich, O. (2010). Vormoor, J. Understanding the cancer stem cell. British Journal of Cancer Vol.103, No. 4, (August 2010), pp. 439 - 445.

Broner, F. \& Carson, M C. Topics in bone biology. Springer. 2009; 4: pp. 2-4. New York, USA.

Buting, K. D. (2002). ABC transporters as phenotypic markers and functional regulators of stem cells. Stem Cells Vol.20, No. 12, (December 2002), pp. 11-20.

Cao, R., Tsukada, Y., Zhang, Y. (2005). Role of Bmi-1 and Ring1A in H2A ubiquitylation and Hox gene silencing. Molecular Cell Vol.22, No. 6, (December 2005),pp. 845-854.

Chen, Y.C., Hsu, H.S., Chen, Y.W., Tsai, T.H., How, C.K., Wang, C.Y., Hung,S.C.,Chang,Y.L.,Tsai,M.L.,Lee,Y.Y.,Ku,H.H.,Chiou,S.H.(2008). $\quad$ Oct-4 expression maintained cancer stem-like properties in lung cancer-derived CD-133 positive cells. PLoS ONE Vol.3, No. 7, (July 2008), pp. 2637.

Dick, J.E. (2003). Stem cells: Self-renewal writ in blood. Nature Vol.423, No. 6937, (May 2003), pp. 231-233.

Du, W., Adam, Z., Rani, R., Zhang, X., Pang, Q. (2008). Oxidative stress in Fanconi anemia hematopoiesis and disease progression. Antioxidants \& Redox Signaling Vol.10, No. 11 (November 2008), pp. 1909-1921.

Frankfurt, O., Licht, J.D., Tallman, M.S. (2007). Molecular characterization of acute myeloid leukemia and its impact on treatment. Current Opinion in Oncology Vol.19, No. 6, (November 2007), pp. 635-649.

Gilliland, D.G.\& Tallman, M.S. (2002). Focus on acute leukemias. Cancer Cell. Vol.1, No. 5, (June 2002), pp. 417-420. 
Gordon, M. Stem cells and haemopoiesis. In: Hoffbrand, V., Catovsky, D., Tuddenham, E.G., $5^{\text {th }}$ ed. Blackwell Publishing, (2005): Differential niche and Wnt requirements during acute myeloid leukemia. pp. 1-12. New York.

Guzman ML, Neering SJ, Upchurch D, Grimes B, Howard DS, Rizzieri DA, Luger SM, Jordan CT (2001). Nuclear factor-KB is constitutively activated in primitive human acute myelogenous leukemia cells. Blood: 2301-2307.

Hanahan, D. \& Weinberg, R.A. (2000). The hallmarks of cancer. Cell Vol.100, No. 1, (January 2000), 57-70.

Hope, K. J., Jin, L., Dick, JE. (2003). Human Acute Myeloid Leukemia Stem Cells. Archives of Medical Research Vol.34, No. 6, (November-December 2003), pp. 507-514.

Hosen, N., Park, C.Y., Tatsumi, N., Oji, Y., Sugiyama, H., Gramatzki, M., Krensky, A.M., Weissman, I.L. (2007). CD96 is a leukemic stem cell-specific marker in human acute myeloid leukemia. Proceedings of National Academy of Science of U S A Vol.104, No. 26, (June 2007), pp. 11008-11013.

Huls, M., Russel, F.G., Masereeuw, R. (2009). The role of ATP binding cassette transporters in tissue defense and organ regeneration. Journal of Pharmacogycal Experimental Therapy Vol.328, No. 1, (January 2009), pp. 3-9.

Jing, D., Fonseca, A.V., Alakel, N., Fierro, F.A., Muller, K., Bornhauser, M., Ehninger, G., Corbeil, D., Ordemann, R. (2010). Hematopoietic stem cells in co-culture with mesenchymal stromal cells-modeling the niche compartments in vitro. Haematologica Vol.95, No. 4, (April 2010), pp. 542550.

Kohler, B.A., Ward, E., McCarthy, B.J., Schymura, M.J., Ries, L.A., Eheman, C., Jemal, A., Anderson, R.N., Ajani, U.A., Edwards, B.K. (2011). Annual report to the nation on the status of cancer, 1975-2007, featuring tumors of the brain and other nervous system. Journal of National Cancer Institute Vol.103, No. 9, (May 2011), pp. 714-736.

Kondo, M. (2010). Lymphoid and myeloid lineage commitment in multipotent hematopoietic progenitors. Immunology Reviews Vol. 238, No. 1, (January 2010), pp. 37-46.

Konopleva, M.Y. \& Jordan, CT. (2011). Leukemia Stem Cells and Microenvironment. Biology and Therapeutic Targeting Vol.29, No. 5, (May 2011), pp. 591-599.

Lane, S.W., Wang, Y.J., Lo Celso, C., Ragu, C., Bullinger, L., Sykes, S.M., Ferraro, F., Shterental, S., Lin, C.P., Gilliland, D.G., Scadden, D.T., Armstrong, S.A., Williams, D.A. (2011). Differential niche and Wnt requirements during acute myeloid leukemia progression. Blood (July 2011), in press.

Lapidot, T, Sirard, C., Vormoor, J., Murdoch, B., Hoang, T., Caceres-Cortes, J., Minden, M., Paterson, B., Caliguri, M.A., Dick, J.E. (1994). A cell initiating human acute myeloid leukaemia after transplantation into SCID mice. Nature Vol.367, No. 6464, (February 1994), pp. 645-648.

Marques, D.S., Sandrini, J.Z., Boyle, R.T., Marins, L.F., Trindade, G.S. (2010). Relationships between multidrug resistance (MDR) and stem cell markers in human chronic myeloid leukemia cell lines. Leukemia Research Vol.34, No. 6, (June 2010), pp. 757762.

Pandolfi, P.P. (2001). In vivo analysis of the molecular genetics of acute promyelocytic leukemia. Oncogene Vol.20, No. 40, (September 2001), pp. 5726-5735.

Pollyea, D.A., Kohrt, H.E., Medeiros, B.C. (2011). Acute myeloid leukaemia in the elderly: a review. British Journal of Haematology Vol.152, No. 5, (March 2011), pp. 524-542. 
Rossi, L., Challen, G.A., Sirin, O., Lin, K.K., Goodell, M.A. (2011). Hematopoietic Stem Cell Characterization and Isolation. Methods in Molecular Biology. Vol.750, No. 2, (2011), pp. 47-59.

Soltanian, S. \& Matin, M. (2011). Cancer stem cells and cancer therapy. Tumor Biology Vol.32, No. 3, (June 2011), pp. 425-440.

Tai, M.H., Chang, C.C., Olson, L.K., Tosko, J.E. (2005). Oct-4 expression in adult human stem cells: evidence in support of the stem cell theory of carcinogenesis. Carcinogenesis Vol.26, No. 2, (February 2005),pp. 495-502.

Tenen, D.G. (2003). Disruption of differentiation in human cancer: AML shows the way. Nature Reviews of Cancer Vol.3, No. 2, (February 2003), pp. 89-101.

Testa, U. (2011). Leukemia stem cells. Annals of Hematology Vol.90, No. 3, (March 2011), pp. 245-271.

Warner, J., Wang, J.C., Hope, K.J., Jin, L., Dick, J.E. (2004). Concepts of human leukemic development. Oncogene Vol.23, No. 43, (September 2004), pp. 7164-7177. 


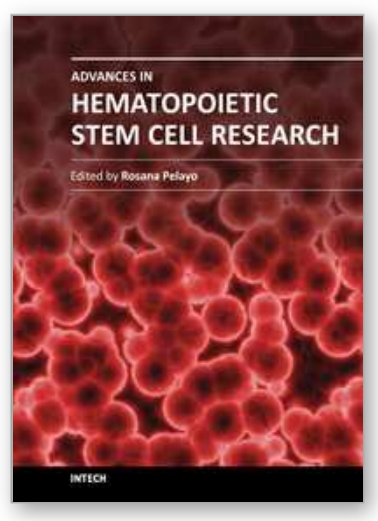

\author{
Advances in Hematopoietic Stem Cell Research \\ Edited by Dr. Rosana Pelayo
}

ISBN 978-953-307-930-1

Hard cover, 464 pages

Publisher InTech

Published online 27, January, 2012

Published in print edition January, 2012

This book provides a comprehensive overview in our understanding of the biology and therapeutic potential of hematopoietic stem cells, and is aimed at those engaged in stem cell research: undergraduate and postgraduate science students, investigators and clinicians. Starting from fundamental principles in hematopoiesis, Advances in Hematopoietic Stem Cell Research assemble a wealth of information relevant to central mechanisms that may regulate differentiation, and expansion of hematopoietic stem cells in normal conditions and during disease.

\title{
How to reference
}

In order to correctly reference this scholarly work, feel free to copy and paste the following:

Sérgio Paulo Bydlowski and Felipe de Lara Janz (2012). Hematopoietic Stem Cell in Acute Myeloid Leukemia Development, Advances in Hematopoietic Stem Cell Research, Dr. Rosana Pelayo (Ed.), ISBN: 978-953-307930-1, InTech, Available from: http://www.intechopen.com/books/advances-in-hematopoietic-stem-cellresearch/hematopoietic-stem-cell-in-acute-myeloid-leukemia-development-

\section{INTECH}

open science | open minds

\section{InTech Europe}

University Campus STeP Ri

Slavka Krautzeka 83/A

51000 Rijeka, Croatia

Phone: +385 (51) 770447

Fax: +385 (51) 686166

www.intechopen.com

\section{InTech China}

Unit 405, Office Block, Hotel Equatorial Shanghai

No.65, Yan An Road (West), Shanghai, 200040, China

中国上海市延安西路 65 号上海国际贵都大饭店办公楼 405 单元

Phone: +86-21-62489820

Fax: +86-21-62489821 
(C) 2012 The Author(s). Licensee IntechOpen. This is an open access article distributed under the terms of the Creative Commons Attribution 3.0 License, which permits unrestricted use, distribution, and reproduction in any medium, provided the original work is properly cited. 